\title{
Elastic vector meson production and DVCS at HERA
}

\author{
Jan Olsson*i \\ DESY, Hamburg, Germany \\ E-mail: jan.olsson@desy.de
}

Recent results from the $\mathrm{H} 1$ and ZEUS experiments are reviewed. The cross sections of elastic photo- and electroproduction of the vector mesons $\phi$ and $J / \psi$ are measured as functions of $Q^{2}, W$ and $t$. The large statistics and the large coverage in $t$ and $W$ allow the determination of the effective Regge exchange trajectories, which are found to be different from the soft pomeron trajectory. Shrinkage is observed in the $J / \psi$ photoproduction.

Helicity analyses are used to determine the spin density matrix element $r_{00}^{04}$ and the $Q^{2}$ dependence of the ratio of the cross sections for longitudinally and transversely polarised photons. S-channel helicity conservation (SCHC) is confirmed for $J / \psi$ production

While the properties of the $\phi$ production put it in a position between "soft" and "hard" diffraction, the $J / \psi$ production shows "hard" features already in photoproduction. All observed properties are consistent with the view of the production process as a photon induced $q \bar{q}$ pair scattering off the proton, thereby forming a vector meson. pQCD-based models are able to give a fair description of the data when at least one of the involved scales reaches a large value. The $J / \psi$ data show some sensitivity to the parameterisation of the gluon distribution in the proton.

Finally, an analysis of Deep Virtual Compton Scattering is reported. The $t$ dependence of the cross section is measured for the first time. Measurements of the $W$ and $Q^{2}$ dependences of the DVCS cross section are also presented. A pQCD-based model with calculations in next-to-leading order describes the data rather well.

International Europhysics Conference on High Energy Physics

July 21st - 27th 2005

Lisboa, Portugal

\footnotetext{
* Speaker.

${ }^{\dagger}$ On behalf of the H1 and ZEUS collaborations
} 


\section{Introduction}

The diffractive, exclusive vector meson (VM) production process,

$$
e p \rightarrow e V Y, \text { with } V=\rho^{\circ}, \omega, \phi, J / \Psi, \Psi^{\prime}, \Upsilon,
$$

and with $Y$ being either a proton (elastic scattering) or a low mass hadronic system (proton dissociative scattering), has been extensively studied at the $e p$ collider HERA. Detailed reviews are given in $[1,2]$. Particular interest is given to the theoretical description of this process in perturbative QCD (pQCD), which can be applied at large values of the physics scales involved, i.e. the squared momentum exchanges $Q^{2}$ and $t$ at the respective photon and proton vertices, and the VM mass $M_{V}$. Thus, exclusive VM production at HERA spans the whole range from "soft" diffraction, described by Regge phenomenology, to "hard" diffraction, where pQCD can be applied.

The QCD description of the diffractive process involves the exchange of several partons, in the simplest form a colour-singlet pair of gluons. While the standard parton distribution functions (PDFs), extracted from the inclusive scattering process, do not contain information about correlations between partons, the process (1.1) in principle offers the possibility to learn more about these correlations, which are encoded in the generalized parton distributions (GPDs)[3]. This is however complicated by the presence of the VM wave function in the final state description. Deep virtual Compton scattering (DVCS), ep $\rightarrow e p \gamma$, where a real photon is produced in diffractive scattering, does not have this complication. The mass difference between the virtual and real photons makes it necessary to consider momentum differences between the gluons in the pair exchange, and this process is currently seen as a promising tool for the measurement and theoretical development of GPDs[4].

The present report describes recent high statistics measurements carried out by the H1 and ZEUS collaborations, of exclusive photo- and electroproduction of the vector mesons $\phi$ and $J / \psi$. A recent measurement of DVCS is also discussed.

\section{Exclusive $\phi$ and $J / \psi$ production}

The ZEUS collaboration has measured the cross section for exclusive $\phi$ electroproduction[5]. In $65 \mathrm{pb}^{-1}$ of data, $\sim 3600$ events in the decay mode $\phi \rightarrow K^{+} K^{-}$were found in the kinematic range $2<Q^{2}<70 \mathrm{GeV}^{2}, 35<W<145 \mathrm{GeV}$ and $|t|<0.6 \mathrm{GeV}^{2}$.

The H1 collaboration presents a recent high statistics study of exclusive photo- and electroproduction of $J / \psi[6]$, at $|t|<1.2 \mathrm{GeV}^{2}$ in $55 \mathrm{pb}^{-1}$ of data. The electroproduction sample, which uses the $\mu^{+} \mu^{-}$decay mode, has the kinematic range $2<Q^{2}<80 \mathrm{GeV}^{2}$ and $40<W<160 \mathrm{GeV}$. In the photoproduction sample, which uses also the $e^{+} e^{-}$decay mode, the $W$ range is $40<W<305$ $\mathrm{GeV}$, due to the use of the backward silicon tracker BST in identifying the $e^{+} e^{-}$decay.

The dependence of the $\phi$ and $J / \psi$ cross sections on these kinematic variables was measured and investigated.

\subsection{The $W$ dependence}

Figure 1 shows the $W$ dependence for the cross sections of $J / \psi$ photo- and electroproduction. The same steep increase with $W$ is seen in photoproduction and electroproduction. The power 
$\delta$, obtained from fits to the form $W^{\delta}$ to the data, is about 0.75 for all values of $Q^{2}$. For the $\phi$ production, corresponding fits in several bins of $Q^{2}$ give lower values $\delta \sim 0.4$, without significant $Q^{2}$ dependence. This can be compared with previous measurements for the light vector mesons $\rho$ and $\omega$, where $\delta \sim 0.2$ has been found. The results confirm the expected increased steepness of the $W$ dependence with increasing quark mass. In this respect, the $\phi$ meson seems to occupy an intermediate position between "soft" and "hard" diffractive production. In QCD, this behaviour of the cross section can be directly related to the increase of the gluon density in the proton at decreasing $x$ (which at any given $Q^{2}$ value corresponds to increasing $W$ ). The present $J / \psi$ data show that the description with QCD models is sensitive to the used gluon distributions[6].
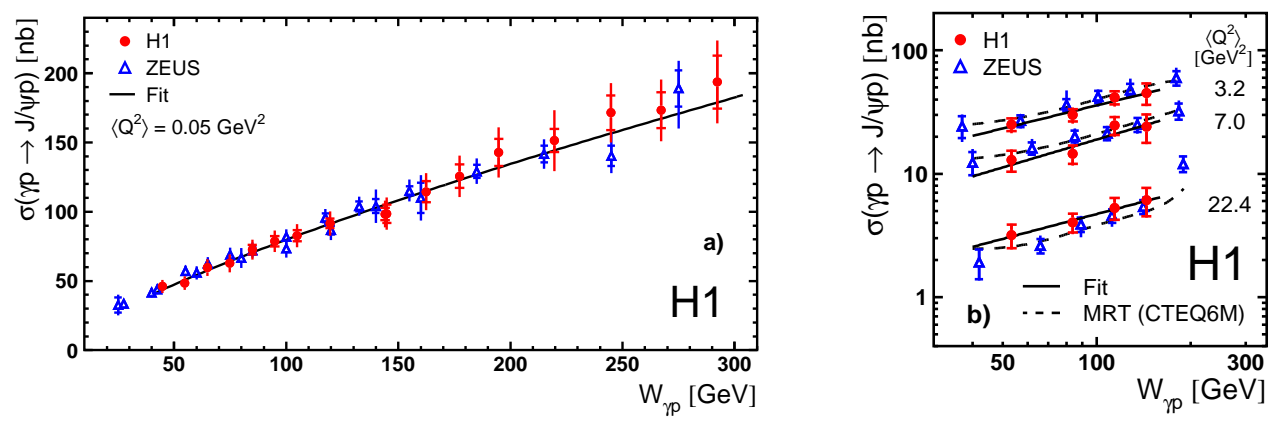

Figure 1: Cross-section of elastic $J / \psi$ production as a function of $W$ in the range $|t|<1.2$ $\mathrm{GeV}^{2}$. The lines are the results of fits to the form $W^{\delta}$ for the $\mathrm{H} 1$ data. (a) Photoproduction, (b) electroproduction, with 3 bins of $Q^{2}$.

\subsection{The $t$ dependence}

In both analyses the $t$ dependence of the cross sections have been fitted to the exponential form $e^{b t}$. For the $\phi$ production values $b \sim 5-6 \mathrm{GeV}^{-2}$ are found, for $J / \psi$ production $b \sim 4 \mathrm{GeV}^{-2}$. No significant $Q^{2}$ dependence is found in either analysis, although the precision of the data does not exclude a decrease of $b$ with increasing $Q^{2}$. These results can be compared with corresponding values for light vector meson production, where $b \sim 7 \mathrm{GeV}^{-2}$ and a decrease of $b$ with increasing $Q^{2}$ is found. Considering that the slope $b$ can be related to the radii of the scattered particles, $b \propto R_{p}^{2} \oplus R_{q \bar{q}}^{2}$, and that $R_{p}^{2} \sim 4 \mathrm{GeV}^{-2}$, the data support the picture of photon-proton diffractive scattering as a process where the photon forms a $q \bar{q}$ pair, which scatters off the proton to form a vector meson. The size of the $q \bar{q}$ pair gets smaller with increasing quark mass (and/or increasing $Q^{2}$ ) and at large values becomes negligibly small compared to the proton size.

\subsection{The combined $W$ and $t$ dependence}

In models based on Regge phenomenology, the energy dependence of the cross section follows a power law, $d \sigma / d t=d \sigma /\left.d t\right|_{t=0, W=W_{0}} \cdot e^{b_{0} t}\left(W / W_{0}\right)^{\delta}$, with $\delta=4(\alpha(t)-1)$. The large statistics in the double differential cross section $d^{2} \sigma / d t d W$ makes it possible to extract the "effective" Regge trajectory $\alpha(t)$ of the exchange. Figure 2a shows the $\phi$ cross section $d \sigma / d|t|$ as a function of $W$ in five bins of $|t|$. Fits to the form $W^{\delta}$ result in the data points $\alpha(\langle t\rangle)$ of figure $2 \mathrm{~b}$. The linear fit $\alpha(t)=$ $\alpha(0)+\alpha^{\prime} \cdot t$ to these data points yields the "effective" Regge trajectory for $\phi$ production, $\alpha(t)=$ $(1.10 \pm 0.2 \pm 0.2)+(0.08 \pm 0.09 \pm 0.08) t$. The value of $\alpha^{\prime}$ is smaller than the "soft pomeron" value 0.25 [7], also shown in figure $2 b$. 

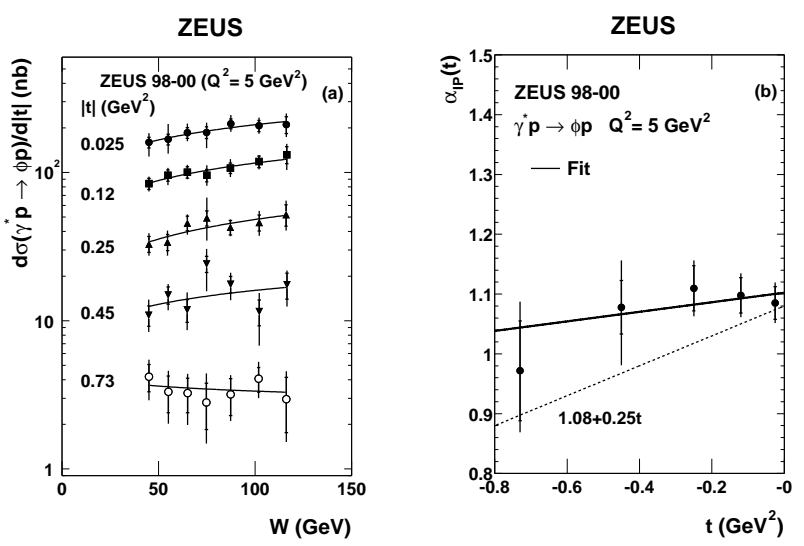

Figure 2: (a) Differential crosssection $d \sigma / d|t|$ as a function of $W$, for five bins of $t$ at $Q^{2}=5 \mathrm{GeV}^{2}$. Full lines are results of fits to the form $W^{\delta}$.

(b) The extracted values of $\alpha(t)$. The full line is a fit of the linear form $\alpha(t)=$ $\alpha(0)+\alpha^{\prime} t$. The dotted line is the soft Pomeron trajectory [7].

In the $\mathrm{H} 1$ analysis of $J / \psi$ production, 1-dimensional fits of the $W$ dependence to the form $W^{\delta}$ are also made in several bins of $t$, for photoproduction and electroproduction data. The resulting values of $\alpha(\langle t\rangle)$ are shown as data-points in figure 3a. 2-dimensional fits of the combined $W$ and $t$ dependence determine the parameters $b_{0}, \alpha(0)$ and $\alpha^{\prime}$ ( $W_{0}$ is fixed to $90 \mathrm{GeV}$, the value is of no consequence for the fits). These fits are also shown in figure $3 \mathrm{a}$, and agree well with the 1-dimensional points. The results are incompatible with the soft pomeron trajectory, both in photoproduction and electroproduction. The latter result, $\alpha(t)=(1.183 \pm 0.0 .054 \pm 0.0 .030)+(0.019 \pm 0.139 \pm$ $0.076) t$, obtained at $\left\langle Q^{2}\right\rangle=8.9 \mathrm{GeV}^{2}$, is within errors compatible with the photoproduction result, $\alpha(t)=(1.224 \pm 0.0 .010 \pm 0.0 .012)+(0.164 \pm 0.028 \pm 0.030) t$.
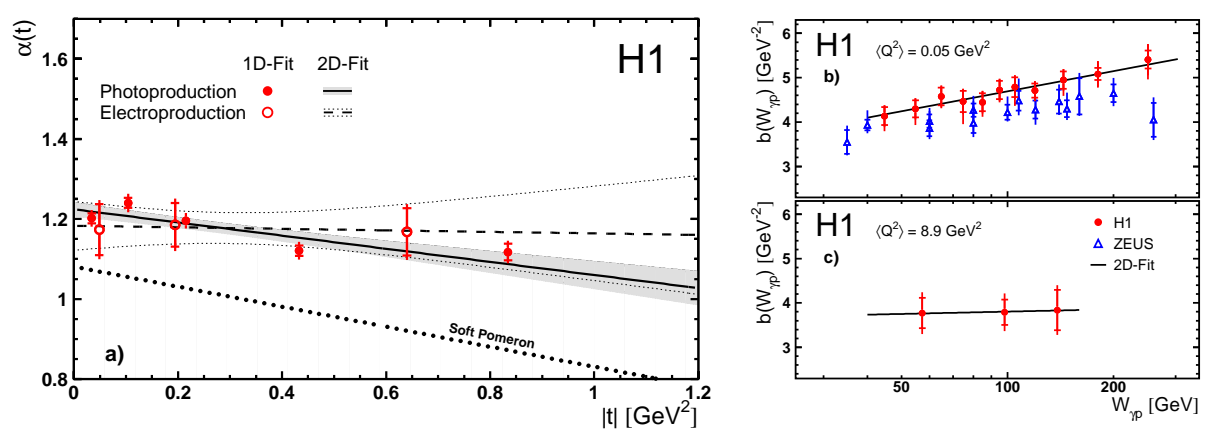

Figure 3: (a) Effective trajectory $\alpha(t)$ as a function of $|t|$ in the range $40<W<305 \mathrm{GeV}$ for photoproduction and $40<W<160 \mathrm{GeV}$ for electroproduction $\left(\left\langle Q^{2}\right\rangle=8.9 \mathrm{GeV}^{2}\right)$. The 1- and 2-dimensional fits are discussed in the text. (b,c) Slope parameter $b(W)$ as a function of $W$ in the range $|t|<1.2 \mathrm{GeV}^{2}$ for (b) photoproduction and (c) electroproduction. Data points are the results of 1-dimensional fits to the form $d \sigma / d t \propto e^{b t}$ in bins of $W$, curves show the 2-dimensional fits.

The parameter $\alpha^{\prime}$ relates the $t$ and $W$ dependences and, if non-zero, leads to "shrinkage" of the diffractive peak with increasing $W$. Significant shrinkage is thus observed for the photoproduction of $J / \psi$. Alternatively, this can be demonstrated from the values of $b=b_{0}+4 \alpha^{\prime} \ln \left(W / W_{0}\right)$, obtained from fits of the $t$ distribution to the form $e^{b t}$ in bins of $W$. These values are shown in figures $3 \mathrm{~b}$ and $3 \mathrm{c}$, with the 2-dimensional fit results (curves) in very good agreement.

\subsection{Angular analysis and the $Q^{2}$ dependence}

The analysis of the angular distributions as a function of $Q^{2}$ gives information on the ratio 
of longitudinal to transverse cross sections, $R=\sigma_{L} / \sigma_{T}$, where the virtual photon-proton cross section $\sigma^{\gamma^{*} p \rightarrow \phi p} \equiv \sigma_{T}+\varepsilon \sigma_{L}$. Under the assumption of S-channel helicity conservation, SCHC (this approximation is valid here, as demonstrated in other, earlier analyses), $R$ can be determined from the spin density matrix element (SDME) $r_{00}^{04}, R=1 / \varepsilon \cdot r_{00}^{04} /\left(1-r_{00}^{04}\right)$, where the flux ratio $\varepsilon$ has a value close to unity in the experimental range. $r_{00}^{04}$ is extracted from the distribution of the polar decay angle $\theta^{*}$ in the $\phi$ or $J / \psi$ rest system, $d \sigma / d \cos \theta^{*} \propto 1+r_{00}^{04}+\left(1-3 r_{00}^{04}\right) \cos ^{2} \theta^{*}$. For the $\phi$ data, figure 4a shows the resulting $Q^{2}$ dependence of $\mathrm{R}$. The $Q^{2}$ dependence of $\sigma_{L}$ and $\sigma_{T}$ for $\phi$ production are shown separately in figure $4 \mathrm{~b}$, with the longitudinal cross section dominating at large values of $Q^{2}$.

In figure 4 two pQCD-based models are compared to the data, both of which give a reasonable description at larger values of $Q^{2}$. The prediction of the FS04 model[8] is somewhat closer to the data than is the MRT prediction[9], although both models fail to describe the data at low $Q^{2}$ values.
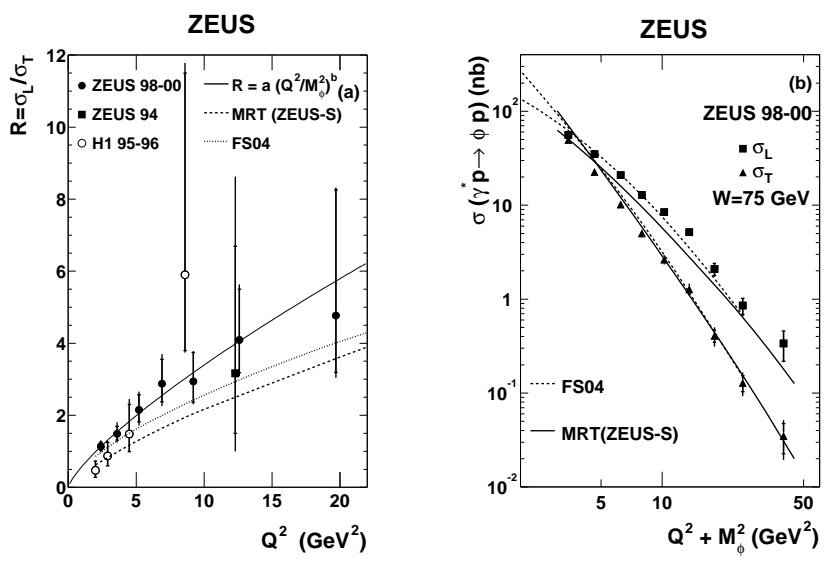

Figure 4: (a) Ratio $R=\sigma_{L} / \sigma_{T}$ as a function of $Q^{2}$, for exclusive $\phi$ production. (b) Longitudinal and transverse parts of the crosssection for exclusive $\phi$ production as a function of $Q^{2}+M_{\phi}^{2}$, for $W=75 \mathrm{GeV}$. Two pQCD-based model predictions $[8,9]$ are also shown.

In the analysis of the $J / \psi$ data further angular distributions were used, to determine beside $r_{00}^{04}$ several more SDMEs. In particular, the relation $r_{1-1}^{1}=\left(1-r_{00}^{04}\right) / 2$, which results from SCHC, was found to hold within experimental errors. Thus the validity of the assumption of SCHC is verified experimentally for exclusive $J / \psi$ production.

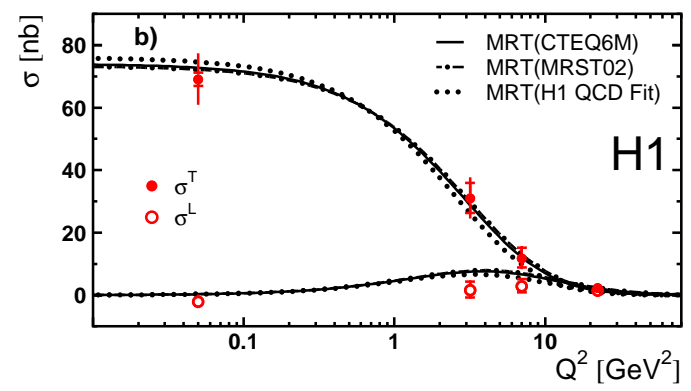

Figure 5: $J / \psi$ production cross sections for longitudinally and transversely polarised photons $\sigma_{L}$ and $\sigma_{T}$ as a function of $Q^{2}$. Curves show MRT[9] QCD calculations with several different gluon distributions.

The cross sections $\sigma_{L}$ and $\sigma_{T}$ for $J / \psi$ production, determined from $r_{00}^{04}$ and $R$ in the same way as for $\phi$ production, are shown in figure 5 as a function of $Q^{2}$. At $Q^{2} \sim M_{J / \psi}^{2}$ they are of similar magnitude, while $\sigma_{T}$ dominates at lower values of $Q^{2}$. The MRT model predictions are compared to the data, with several different gluon distribution parameterisations, and are in good agreement. 


\section{Deep Virtual Compton Scattering, DVCS}

The H1 collaboration presents an analysis of the DVCS process[10], using two $e^{+} p$ data samples corresponding altogether to $\sim 46 \mathrm{pb}^{-1}$. The kinematic range of the $\sim 1240$ events is $2<Q^{2}<80 \mathrm{GeV}^{2}$ and $4<Q^{2}<80 \mathrm{GeV}^{2}$ (due to different trigger conditions in the two samples), $3<W<140 \mathrm{GeV}$ and $|t|<1 \mathrm{GeV}^{2}$. The final state of the DVCS reaction, $e^{+}+p \rightarrow e^{+}+p+\gamma$, consists only of a photon and a positron (the scattered proton is not detected). It is indistinguishable from the Bethe-Heitler (BH) bremsstrahlung process, which constitutes an important background. Two samples are therefore used: the DVCS sample, where the scattered positron is detected in the backward calorimeter SpaCal and the photon in the Liquid Argon calorimeter (LAr), and the BH sample, where the positron is detected in LAr and the photon in SpaCal. The latter sample occupies a phase space region where the DVCS process is much suppressed, and can thus be used to normalise the bremsstrahlung background, which can be exactly calculated in both phase space regions. Since the interference term in the total cross section, $\sigma=\sigma_{D V C S}+\sigma_{B H}+I_{B H, D V C S}$, is $\sim 0$ due to the fact that the angle between the positron and proton in the transverse scattering plane is integrated over, the DVCS cross section can be measured by subtracting the BH cross section from the total cross section in the relevant phase space region.
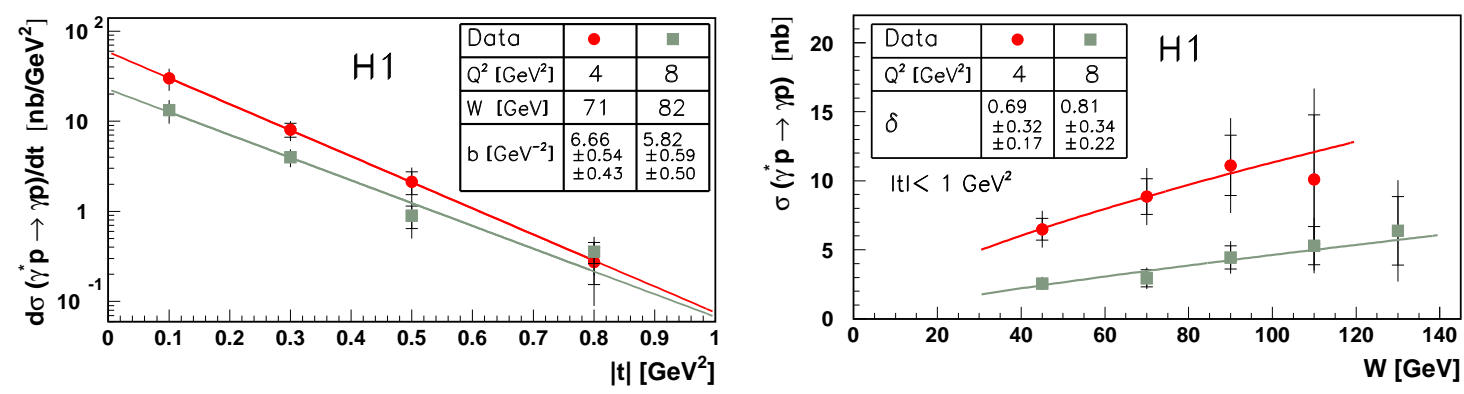

Figure 6: (a) DVCS cross-section differential in $t$, for two values of $Q^{2}$ and $W$. Lines show the results of fits to the form $e^{-b|t|}$. (b) DVCS cross-section differential in $W$, for two values of $Q^{2}$ and with $|t|<1 \mathrm{GeV}^{2}$. Lines show the results of fits to the form $W^{\delta}$.

The $t$ dependence of the cross section, shown in figure 6a for 2 values of $\left\langle Q^{2}\right\rangle$, is here measured for the first time. Fitting to the form $e^{b t}$, the combined value for the slope at $\left\langle Q^{2}\right\rangle=8 \mathrm{GeV}^{2}$ is obtained, $b=6.02 \pm 0.35 \pm 0.39 \mathrm{GeV}^{-2}$. For model calculations this is an important measurement, since proper normalisation is now possible (previously an assumed value of $b=7 \pm 2 \mathrm{GeV}^{-2}$ was used).

The $W$ dependence of the DVCS cross section is shown in figure $6 \mathrm{~b}$. Fitting to the form $W^{\delta}$, the combined value is obtained, $\delta=0.77 \pm 0.23 \pm 0.19$. The steep $W$ dependence is thus similar to the behaviour observed for $J / \psi$ production, while the $t$ dependence is similar to the behaviour observed for light vector mesons.

pQCD calculations have been performed in next-to-leading order (NLO), using $Q^{2}$ as hard scale. As mentioned above, generalized parton distributions have to be considered. One such calculation[11] is shown in figure 7, for two different PDFs. In the model calculations, these PDFs are used as starting point, and the momentum difference for the two exchanged gluons is generated dynami- 
cally. As seen in figure 7, a fair description of the cross section is given, both in the $Q^{2}$ dependence and in the $W$ dependence.

Alternative models (not shown), based on a colour dipole picture of the scattering[12, 13, 14], are also able to describe the data well.
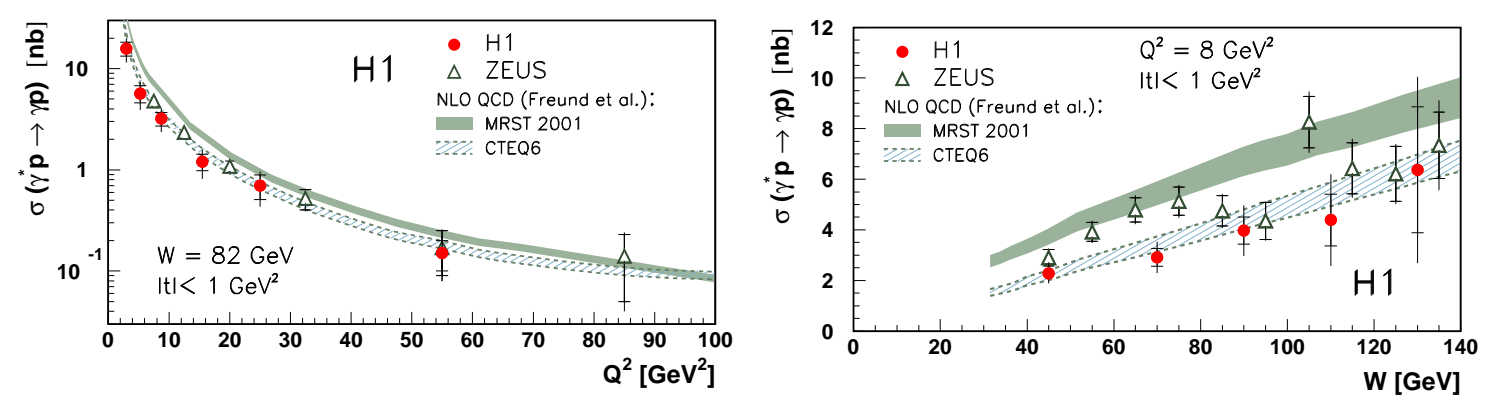

Figure 7: (a) DVCS cross-section differential in $Q^{2}$, for $W=82 \mathrm{GeV}$ and $|t|<1 \mathrm{GeV}^{2}$. (b) DVCS cross-section differential in $W$, for $Q^{2}=8 \mathrm{GeV}^{2}$ and $|t|<1 \mathrm{GeV}^{2}$. Curves show QDC predictions at NLO by Freund et al.[11], based on MRST 2001 and CTEQ6 PDFs.

\section{Acknowledgements}

It is a pleasure to thank the organizers for an exciting and well prepared conference. I wish to thank my colleagues in $\mathrm{H} 1$ and ZEUS for providing the results shown in this report and for all their help given to me.

\section{References}

[1] J.A. Crittenden, Springer Tracts in Modern Physics, Volume 140 (Springer, Berlin Heidelberg, 1997)

[2] H. Abramowicz and A. Caldwell, Rev.Mod.Phys. 71 (1999) 1275 [hep-ex/9903037].

[3] M. Diehl, Phys.Rep. 388 (2003) 41 [hep-ph/ 0307382 ].

[4] X. Ji, Phys.Rev.D55 (1997) 7114 [hep-ph / 9609381$]$;

A.V. Radyushkin, Phys.Rev.D58 (1998) 114008 [hep-ph/ 9803316$]$;

J.C. Collins and A. Freund, Phys.Rev.D59 (1999) 074009 [hep-ph/ 9801262$].$

[5] ZEUS Collaboration, S. Chekanov et al., Nuclear Physics B 718 (2005) 3 [hep-ex/ 0504010 ].

[6] H1 Collaboration, A. Aktas et al., subm. to Eur.Phys.J [hep-ex/0510016].

[7] A. Donnachie and P.V. Landshoff, Phys.Lett.B 348 (1995) 213.

[8] J.R. Forshaw and G. Shaw, [hep-ph/0411337].

[9] A.D. Martin, M.G. Ryskin and T. Teubner, Phys.Rev.D 62 (2000) 014022 [hep-ph/9912551].

[10] H1 Collaboration, A. Aktas et al., Eur.Phys.J C 44 (2005) 1 [hep-ex/ 0505061 ].

[11] A. Freund, M.F. McDermott and M. Strikman, Phys.Rev. D 67 (2003) 036001 [hep-ph/ 0208160 ].

[12] A. Donnachie and H.G. Dosch, Phys.Lett.B502 (2001) 74 [hep-ph/ 0010227 ].

[13] L. Favart and M.V. Machado, Eur.Phys.J. C29 (2003) 365 [hep-ph/0302079].

[14] L. Favart and M.V. Machado, Eur.Phys.J. C34 (2004) 429 [hep-ph/ 0402018 ]. 